\title{
Performance Analysis of a High-Speed All-Optical Subtractor using a Quantum-Dot Semiconductor Optical Amplifier-Based Mach-Zehnder Interferometer
}

\author{
Mohammad Reza Salehi* and Seyed Farhad Taherian \\ Department of Electrical and Electronics Engineering, Shiraz University of Technology, Shiraz, Iran
}

(Received November 28, 2013 : revised February 4, 2014 : accepted February 4, 2014)

\begin{abstract}
This paper presents the simulation and design of an all-optical subtractor using a quantum-dot semiconductor optical amplifier Mach-Zehnder interferometer (QD-SOA MZI) structure consisting of two cascaded switches, the first of which produces the differential bit. Then the second switch produces the borrow bit by using the output of the first switch and the subtrahend data stream. Simulation results were obtained by solving the rate equations of the QD-SOA. The effects of QD-SOA length, peak power and current density have been investigated. The designed gate can operate at speeds of over $250 \mathrm{~Gb} / \mathrm{s}$. The simulation results demonstrate a high extinction ratio and a clear and wide-opening eye diagram.
\end{abstract}

Keywords: All-optical logic, Mach-Zehnder interferometer, Quantum dot SOA, Optical subtractor

OCIS codes : (200.4660) Optical logic; (250.5980) Semiconductor optical amplifiers; (320.0320) Ultrafast optics

\section{INTRODUCTION}

All-optical gates will be essential elements in future communications systems. All-optical processing has many applications, such as wavelength conversation, add-drop multiplexing, regeneration clock recovery, simple bit-pattern recognition, address recognition, signal processing, and packet synchronization [1-3]. Subtraction is one of the most important operations in Boolean functions and is used in binary subtraction, binary counting, arithmetic logic units, encryption, and decryption in security networks. Many designs for a single subtractor or subtractor with adder have been proposed by using a semiconductor optical amplifier (SOA) and periodically-poled lithium niobate (PPLN) waveguide [4], micro-ring resonator [5], exploiting four-wave mixing (FWM) in a SOA [6], a SOA based-Mach-Zehnder interferometer (MZI) [7], a three-state system [8], a terahertz optical asymmetric demultiplexer (TOAD) [9], a single-slot waveguide [10], two SOAs [11], implementing nonlinear material [12], and a nonlinear directional coupler [13]. SOA with quantum-dot active region is a promising candidate for ultrafast operations [14-15]. The high output power, low threshold current density, ultra-fast response behavior, good temperature stability, and low noise level of the quantum-dot SOA (QD-SOA) have been demonstrated, and it has been emphasized that these elements can be utilized as building blocks of alloptical systems [17-19]. In this paper, using two QD-SOA MZI, we design a subtractor that can operate and perform well at speeds of over $250 \mathrm{~Gb} / \mathrm{s}$.

\section{REALIZATION OF ALL-OPTICAL SUBTRACTOR}

The subtractor is a combinational logic device that performs subtraction between two binary numbers. Its $\mathrm{A}$ and $\mathrm{C}$ inputs are for the minuend and subtrahend respectively. The differential and borrow outputs are $\mathrm{D}$ and $\mathrm{B}$ respectively. If $\mathrm{A}$ and $\mathrm{C}$ are equal then $\mathrm{B}=\mathrm{D}=0$, but if $\mathrm{A}=1$ and $\mathrm{C}=0$ then the outputs will be $\mathrm{D}=1, \mathrm{~B}=0$. If $\mathrm{A}=0$ and $\mathrm{C}=1$ the outputs will be $\mathrm{D}=1$ and $\mathrm{B}=1$. Figure 1 shows the structure of our proposed subtractor. In the first MZI a differential bit is generated for which XOR operation has been done between $\mathrm{A}$ and $\mathrm{C}$. Next the borrow bit is generated in the second MZI in which the differential bit acts as a probe signal. The theoretical results were verified by numerical simulation.

The output powers of the gate are expressed as,

\footnotetext{
*Corresponding author: salehi@sutech.ac.ir

Color versions of one or more of the figures in this paper are available online.
} 


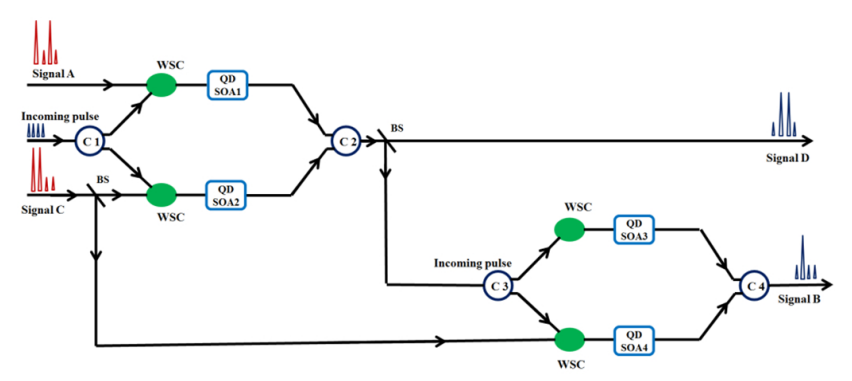

FIG. 1. Configuration of an all-optical subtractor based on QD-SOA MZI. C1, C2, C3, C4: (ideally 50:50) 3 dB couplers, BS: beam splitter, WSC: wavelength-selective coupler, QD-SOA: quantum-dot SOA.

$$
\begin{aligned}
P_{\text {Differential }}(\mathrm{t}) & =\frac{\operatorname{Pin}(\mathrm{t})}{8}\left\{G_{1}(t)+G_{2}(t)-2 \sqrt{G_{1}(t) \times G_{2}(t)}\right. \\
& \left.\times \cos \left[-\frac{\alpha}{2} \ln \left(\frac{G_{1}(t)}{G_{2}(t)}\right)\right]\right\} \\
P_{\text {Borrow }}(\mathrm{t}) & =\frac{P_{\text {Differential }}(\mathrm{t})}{4}\left\{G_{3}(t)+G_{4}(t)-2 \sqrt{G_{3}(t) \times G_{4}(t)}\right. \\
& \left.\times \cos \left[-\frac{\alpha}{2} \ln \left(\frac{G_{3}(t)}{G_{4}(t)}\right)\right]\right\}
\end{aligned}
$$

where Pin ( $\mathrm{t}$ ) denotes probe power that is a continuous wave pulse, $G_{1,2,3,4}(t)$ are the instantaneous gains of $Q D-S O A_{1,2,3,4}$ respectively, and $\alpha$ is the QD-SOA linewidth enhancement factor. To calculate gain and power we must solve the three-level rate equation for the electron transmission between the wetting layer (WL), exited state (ES), and ground state (GS) respectively, which are as follows [20-22].

$$
\begin{aligned}
& \frac{\partial N_{W}}{\partial t}=\frac{J}{e L_{w}}-\frac{N_{W}(1-h)}{\tau_{w 2}}+\frac{N_{Q} h}{L_{w} \tau_{2 w}}-\frac{N_{W}}{\tau_{w R}} \\
& \frac{\partial h}{\partial t}=\frac{L_{w} N_{W}(1-h)}{N_{Q} \tau_{w 2}}+\frac{f(1-h)}{\tau_{12}}-\frac{h}{\tau_{2 w}}-\frac{(1-f) h}{\tau_{21}} \\
& \frac{\partial f}{\partial t}=-\frac{g_{\text {max }}(2 f-1) L_{w} V_{g} S}{N_{Q}}-\frac{f(1-h)}{\tau_{12}}-\frac{f^{2}}{\tau_{1 R}}+\frac{(1-f) h}{\tau_{21}}
\end{aligned}
$$

and the photon rate equation is

$$
\frac{\partial S}{\partial \mathrm{Z}}=g_{\text {max }}(2 f-1) S-\alpha_{i n t} S
$$

where $\mathrm{z}$ is the longitudinal direction along the QD-SOA length $\mathrm{L}, \mathrm{t}$ is the local time, and $V_{g} \approx 8.3 \times 10^{7} \mathrm{~m} / \mathrm{s}$, $S(z, t)=P(z, t) /\left(A_{\text {eff }} V_{g} h v\right)$ is the photon density of the input data signal. $\mathrm{g}_{\max }$ is maximum modal gain, $\alpha_{\text {int }}$ is material absorption coefficient, $h v$ is the photon energy, $\mathrm{J}$ is the injection current density, e is electron charge, $N_{W}$ is the electron density in WL, and $\mathrm{h}$ and $\mathrm{f}$ are the electron occupation probabilities in the ES and GS respectively. $N_{Q}$ is the surface density of QDs, $L_{W}$ is the effective thickness of the active layer, $\tau_{2 w}$ is the electron escape time from the ES to the WL, $\tau_{W R}$ is the spontaneous radiative life time in $\mathrm{WL}, \tau_{21}$ is the electron relaxation time from the GS to the ES, and $\tau_{1 R}$ is the spontaneous relaxation life time in the QDs.

\section{SIMULATION RESULTS AND DISCUSSION}

In order to investigate the feasibility of the proposed design, we have applied the 4th-order Runge-Kutta method to solve equations (3-5) numerically and to calculate the gain coefficient. The input pulses are pseudo-random binary sequences at $250 \mathrm{~Gb} / \mathrm{s}$ whose profile is Gaussian, that is $P(\mathrm{t})=P_{\text {max }} \exp \left[-4 \operatorname{Ln}\left(\frac{t}{T_{F W H M}}\right)^{2}\right]$, where $P_{\max }$ is peak power and $T_{F W H M}$ is their full width at half maximum and less than 1.5 ps. By using Leibniz's integral rule, we can interchange the integral and partial differential operators. By employing Leibniz's rule for $S(\mathrm{z}, \mathrm{t})$, we have

$$
\int_{0}^{L} S_{t}(\mathrm{z}, \mathrm{t}) d z=\frac{d}{d t}\left(\int_{0}^{L} S(\mathrm{z}, \mathrm{t}) d z\right)
$$

and by integrating along the $z$ variable in equation (7) we have

$$
L S_{t}(\mathrm{z}, \mathrm{t}) d z=\frac{d}{d t}\left(\int_{0}^{L} S(\mathrm{z}, \mathrm{t}) d z\right)
$$

The next integration along the $t$ variable in equation (8) gives

$$
\bar{S}(t)=\frac{1}{L}\left(\int_{0}^{L} S(\mathrm{z}, \mathrm{t}) d z\right)
$$

where $\bar{S}(t)$ is the average of $S(\mathrm{z}, \mathrm{t})$ along the $\mathrm{t}$ variable. By integrating and normalizing $[0, z]$, and solving for the optical power at location $\mathrm{z}$ in terms of the input power for $S(\mathrm{z}, \mathrm{t})$ in equation (6), and using equation (9), we have:

$$
\bar{S}(t)=\frac{S(0, \mathrm{t})\left(\exp \left(\mathrm{g}-\alpha_{\text {int }}\right) \mathrm{L}-1\right)}{\left(\mathrm{g}-\alpha_{\text {int }}\right) \mathrm{L}}
$$

where $g=g_{\max }(2 f-1)$. In addition, the gain is calculated 
from $G(\mathrm{t})=\frac{S(\mathrm{~L}, \mathrm{t})}{S(0, \mathrm{t})}=\exp \left(\left(\mathrm{g}-\alpha_{\text {int }}\right) \mathrm{L}\right)$. The simulation results and their comparison to the results of other papers verify this method. It was found that our method can solve rate equations more quickly than previous methods [18, 19]. The extinction ratio of the input signals was assumed to be $10 \mathrm{~dB}$. The parameter values were taken from the literature on other QD-SOA-based interferometric gates [19-22]. These are: $g_{\max }=14 \mathrm{~cm}^{-1}, N_{Q}=5 \times 10^{10} \mathrm{~cm}^{-2}, \tau_{W 2}$ $=3 \mathrm{ps}, \tau_{2 W}=1 \mathrm{~ns}, L_{W}=0.25 \mu \mathrm{m}, \tau_{21}=0.16 \mathrm{ps}, \tau_{1 R}=0.4 \mathrm{~ns}$, $\tau_{12}=1.2 \mathrm{ps}, L=4 \mathrm{~mm}, P_{\max }=10 \mathrm{dBm}, \alpha=2$ and $J=2.5 \mathrm{kA} / \mathrm{cm}^{2}$. In order to assess the performance of an optical logic design, the most important parameters are extinction ratio, amplitude modulation, quality factor, pseudo-eye diagram, and relative eye opening, which we will investigate. The extinction ratio (ER) is defined as

$$
E R(\mathrm{~dB})=10 \log \left(\frac{P_{\min }^{1}}{P_{\max }^{0}}\right)
$$

where $P_{\min }^{1}$ and $P_{\max }^{0}$ are the minimum and maximum values of the peak power of " 1 " and " 0 ", respectively [23]. In order to distinguish 1 unambiguously from 0 the ER must be over $10 \mathrm{~dB}$ [23]. Figure 2(a) depicts the ER variation versus QD-SOA length for three different peak power values of input data signals, where the other parameters are kept fixed. It is observed in the curves that for $4<L<5 \mathrm{~mm}, E R>14.5 \mathrm{~dB}$ will result. The maximum values are shifted to the right as the QD-SOA length becomes larger. The smaller QD-SOA lengths need stronger control signals. Figure 2(b) shows the variation of ER with peak power for three different current densities. This shows that ER decreases with increasing peak power and increases with larger current density. Figure 2(c) shows the ER variation with current density for three different QD-SOA lengths. For $J>2 \mathrm{kA} / \mathrm{cm}^{2}$ we have $E R>16 d B$ because at higher injected current density there are more carriers in the wetting layer, and we have a better response.

The ratio between maximum and minimum peak power for signals corresponding to " $1 "$ is defined as

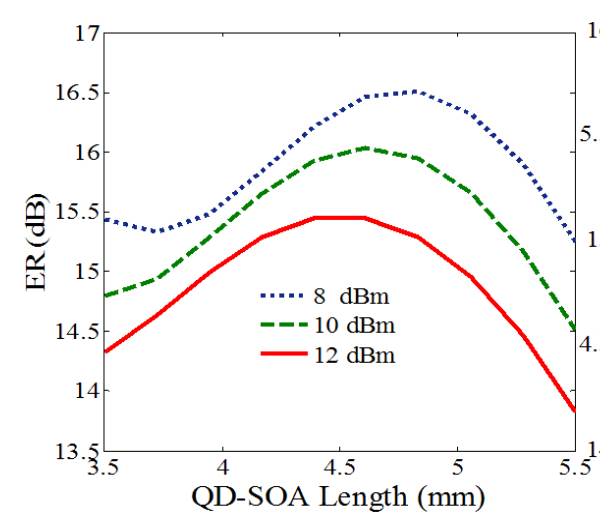

(a)

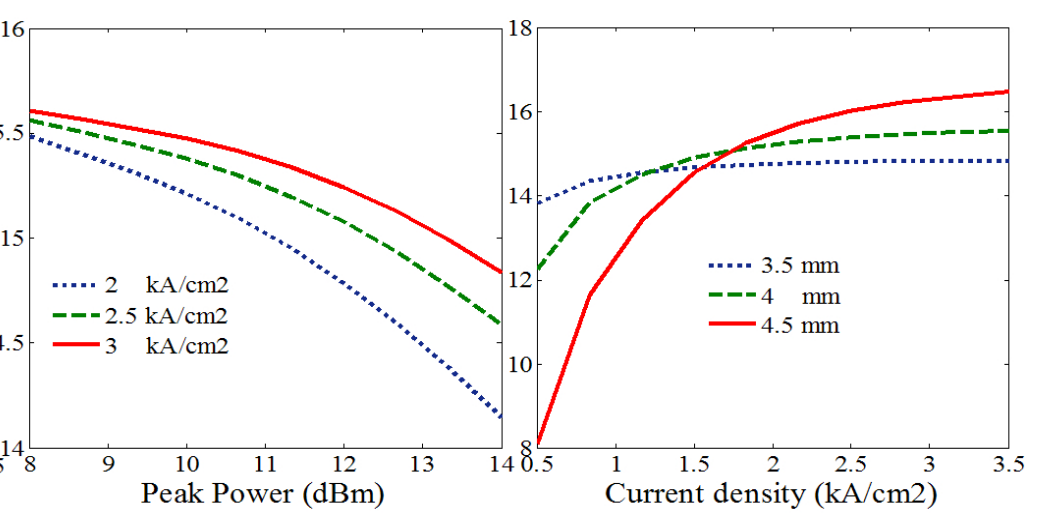

(b)

(c)

FIG. 2. ER variation versus (a) QD-SOA length for three different peak powers, (b) peak power for three different current densities, and (c) current density for three different lengths.

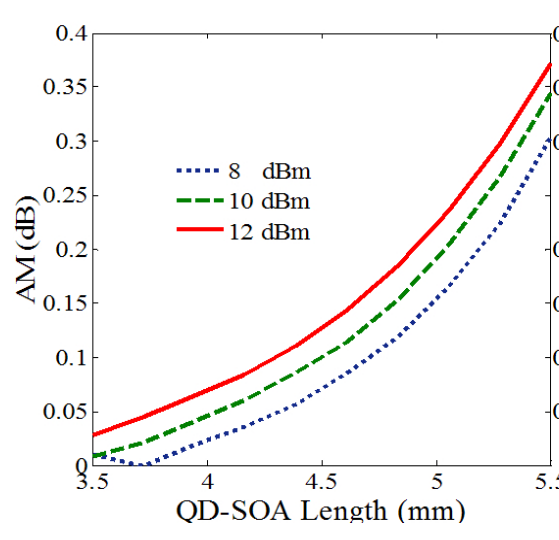

(a)

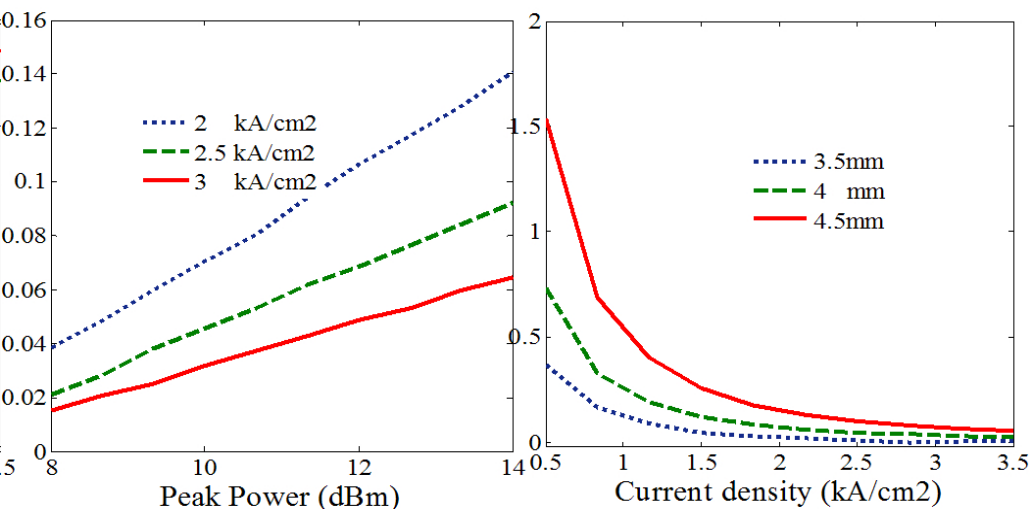

(b)

(c)

FIG. 3. AM variation versus (a) QD-SOA length for three different peak powers, (b) peak power for three different current densities, and (c) current density for three different lengths. 


$$
A M(\mathrm{~dB})=10 \log \left(\frac{P_{\max }^{1}}{P_{\min }^{1}}\right)
$$

where $P_{\max }^{1}$ and $P_{\min }^{1}$ is the maximum peak power of "1" and "1" respectively [24]. The lower the AM, the more uniform is the level of the "1" outputs, and the smaller is the related pattern effect. Figure 3(a) illustrates the effect of QD-SOA length on the AM for three different peak powers of input data signals. For $L<4 \mathrm{~mm}$ a very small $\mathrm{AM}$ is obtained, which is very desirable, and $\mathrm{AM}$ increases with larger peak powers. In Fig. 3(b) variations of AM with peak power for three different current densities are shown. The peak power increase has an undesirable effect on AM, while the increase of current density has a desirable effect on it. Figure 3(c) shows the variation of $\mathrm{AM}$ with $\mathrm{J}$ for three different QD-SOA lengths. It is observed that for $J>2 \mathrm{kA} / \mathrm{cm}^{2}$ we can achieve a small $\mathrm{AM}$, and that $\mathrm{AM}$ increases with increasing of length.

The quality factor is defined as

$$
Q(\mathrm{~dB})=\left(\frac{m_{1}-m_{0}}{\sigma_{1}+\sigma_{0}}\right)
$$

where $m_{1}\left(m_{0}\right)$ and $\sigma_{1}\left(\sigma_{0}\right)$ are the average power and standard deviation of the circuit output at " 1 " ("0") respectively [25]. Figure 4 shows that Q decreases for $L>4.5 \mathrm{~mm}$ and a larger peak power results from the decrease in $\mathrm{Q}$ factor. The results show that with $J>$ $2.5 \mathrm{kA} / \mathrm{cm}^{2}$ and $L=4 \mathrm{~mm}$ we can achieve a larger Q.

A pseudo-eye diagram is one of the best standards for assessing the output signals. A clear and open eye diagram is desired. Figure 5 illustrates the effect of electron relaxation time from ES to GS on PED. A slow transition of the electron between ES and WL results in a slower cross gain modulation. Thus speed is limited by the relaxation time from the wetting layer to the quantum-dot state.

The relative eye opening is defined as

$$
\text { eye - opening }(\%)=\left(\frac{P_{\min }^{1}-P_{\max }^{0}}{P_{\min }^{1}}\right)
$$

where $P_{\min }^{1}$ and $P_{\max }^{0}$ are the minimum and maximum powers for ones and zeros respectively [10]. In this design the related eye opening is over $90 \%$. Table 1 shows the relative eye opening for three QD-SOA lengths and three electron relaxation times from ES to GS. The other

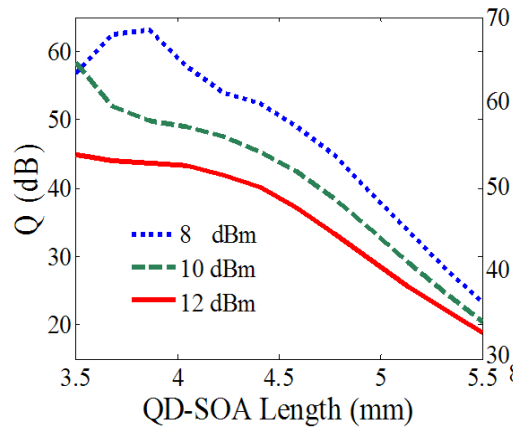

(a)

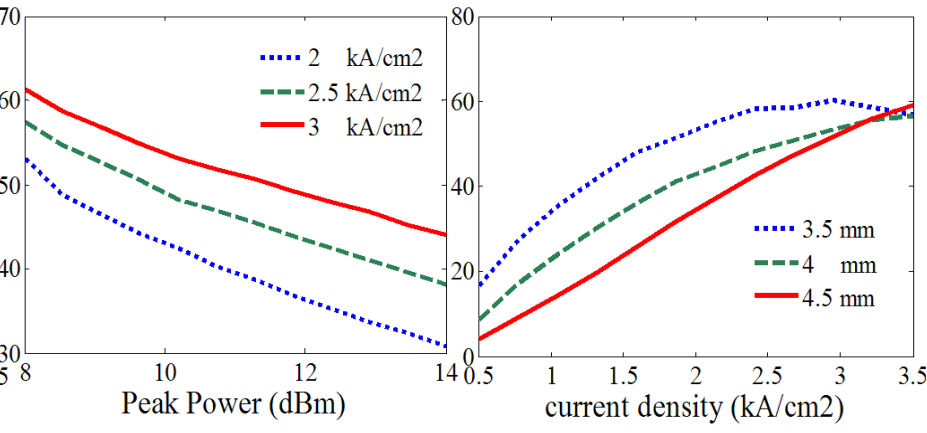

(b) (c)

FIG. 4. Q variation versus (a) QD-SOA length for three different peak powers, (b) peak power for three different current densities, and (c) current density for three different lengths.

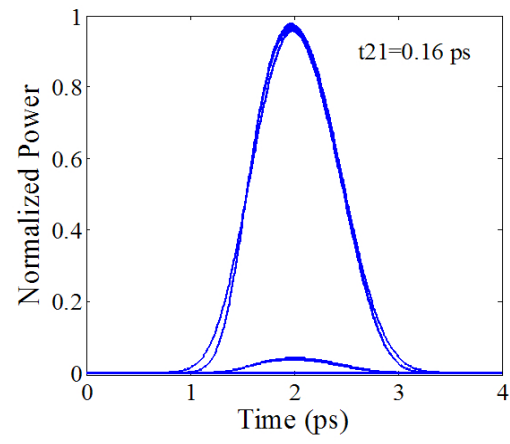

(a)

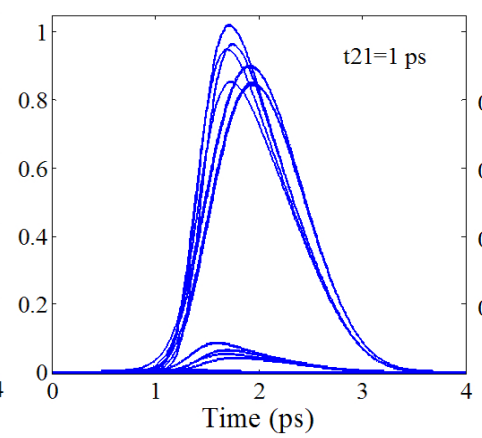

(b)

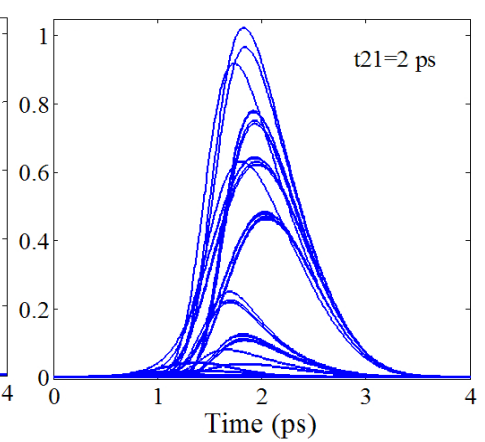

(c)

FIG. 5. Simulated pseudo-eye diagrams of output waveforms for electron relaxation time from the ES to the GS (a) $0.16 \mathrm{ps,} \mathrm{(b)} 1$ ps, and (c) 2 ps. 
parameters are kept fixed.

According to Figs. 2-5 and our discussions so far, QDSOAs with lengths smaller than $4.5 \mathrm{~mm}$, show desirable responses but smaller lengths decrease unsaturated gain. Table 2 shows gain values for five different QD-SOA lengths. It was found that with current density of more than $2.5 \mathrm{kA} / \mathrm{cm}^{2}$ we can achieve a more suitable performance. Increasing the current density might not be practical for a QD-SOA [22], however, and we see that with smaller peak power the output is better, so for good switching we choose a peak power of $10 \mathrm{~dB}$.

According to the above mentioned explanations and using the combination of input parameters $L=4 \mathrm{~mm}, J$ $2.5 \mathrm{kA} / \mathrm{cm}^{2}, P_{\max }=10 \mathrm{dBm}$ and $\tau_{21}=0.16 \mathrm{ps}$, we can achieve these values in the output: $\mathrm{ER}=15.37 \mathrm{~dB}, \mathrm{AM}=0.052$ $\mathrm{dB}$, eye-opening $=97.09 \%, \mathrm{Q}=49.13 \mathrm{~dB}$, and $20.82 \mathrm{~dB}$ of unsaturated gain for $250 \mathrm{~Gb} / \mathrm{s}$ input signals. Figure 6 shows input and output data streams for a 32-bit pseudorandom binary sequence of input.

Table 3 illustrates the output parameters of the proposed structure, compared to those of similar recent structures. The last column shows the parameters of our designed structure, which demonstrates its better performance, due to our choice of optimal parameters for the structure, and use of the transmitting ports in both MZIs.

The main reason for the faster response of a QD-SOA compared to a bulk SOA is the presence of the wetting layer. The wetting layer serves as a carrier reservoir: Carriers depleted by the injected optical pulse in the QD

TABLE 1 . Variations of relative eye opening with $L$ and $\tau_{21}$

\begin{tabular}{c|c|c|c|c|c|c}
\hline \hline Parameter & $\mathrm{L}=2 \mathrm{~mm}$ & $\mathrm{~L}=4 \mathrm{~mm}$ & $\mathrm{~L}=6 \mathrm{~mm}$ & $\tau_{21}=0.16 p s$ & $\tau_{21}=1 p s$ & $\tau_{21}=2 p s$ \\
\hline Eye-O (\%) & 98.9 & 97.09 & 94.74 & 97.09 & 95.2 & 83.67 \\
\hline
\end{tabular}

TABLE 2. Variation of QD-SOA gain with length

\begin{tabular}{c|c|c|c|c|c}
\hline \hline 4 & 3.75 & 3.5 & 3.25 & 3 & $\mathrm{~L}(\mathrm{~mm})$ \\
\hline 120 & 90 & 67 & 50 & 37 & Gain \\
\hline
\end{tabular}

(a)

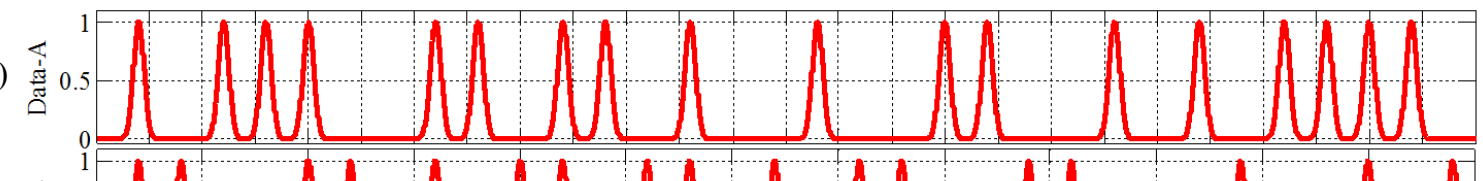

(b)

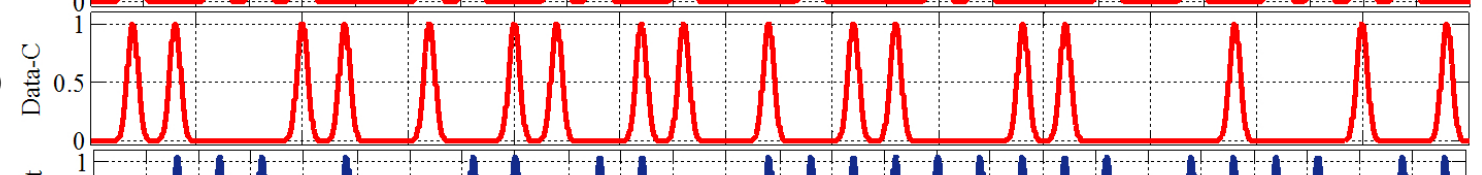

(c)

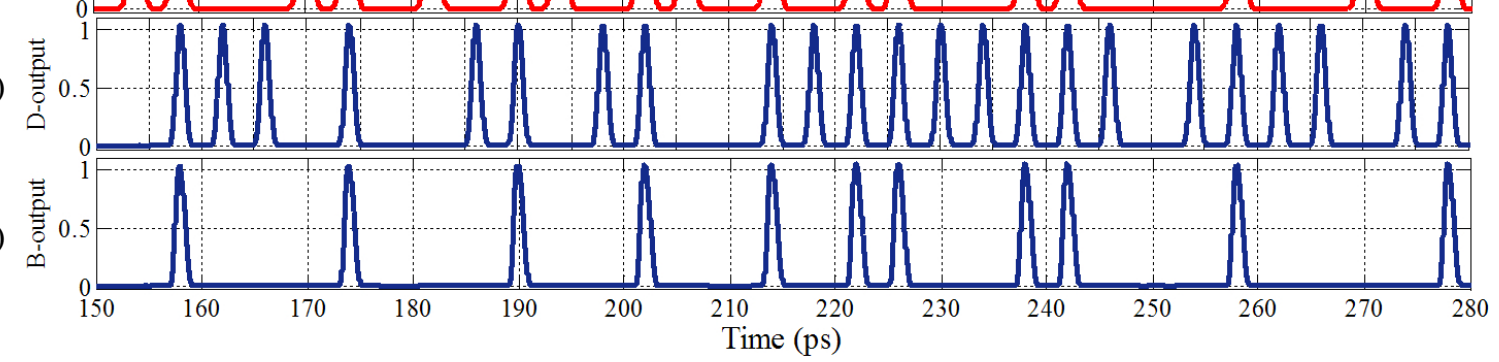

FIG. 6. Input and output waveforms of the all-optical subtractor. (a) minuend input, (b) subtrahend input, (c) differential output, and (d) borrow output.

TABLE 3. The comparison of parameters of the proposed optical gate with those of other papers

\begin{tabular}{c|c|c|c|c|c|c}
\hline \hline paper & {$[16]$} & {$[21]$} & {$[23]$} & {$[24]$} & {$[10]$} & This work \\
\hline Gate & NOR & Latch & NOT & NAND & subtractor & subtractor \\
\hline Speed $(\mathrm{Gb} / \mathrm{s})$ & 250 & 250 & 160 & 160 & 160 & 250 \\
\hline ER $(\mathrm{dB})$ & - & - & 12 & - & 10 & 15.37 \\
\hline Q $(\mathrm{dB})$ & 17.33 & 10 & - & - & 20 & 49.13 \\
\hline AM $(\mathrm{dB})$ & - & - & - & 0.5 & - & 0.052 \\
\hline
\end{tabular}


ground state are replaced from the wetting layer by fast carrier transfer. At a higher injected current density there are more carriers in the wetting layer, resulting in a better performance. Thus the speed of optical logic gates is limited by the carrier recovery time in the device. Careful adjustment of the peak power of the modulated signal and QD-SOA bias current is necessary for error-free performance at high data rates and for avoiding overlap in response.

\section{CONCLUSION}

The effects of the most important parameters on the performance of the proposed gate are investigated using a model of three coupled equations for a QD-SOA into which a Gaussian pulse with rate $250 \mathrm{~Gb} / \mathrm{s}$ has been inserted. The results show that output signals have high ER and Q and low AM, and that the gate can operate well at speeds of over $250 \mathrm{~Gb} / \mathrm{s}$. In both MZIs we used the transmitting port. The reflecting port can also be used to extend the device in generating other logic gates.

\section{REFERENCES}

1. A. Dolatabady and N. Granpaye, "All optical logic gates based on two dimensional plasmonic waveguides with nanodisk resonators," J. Opt. Soc. Korea 16, $432-442$ (2012).

2. S. Kaur and R.-S. Kaler, "Ultrahigh speed reconfigurable logic operations based on single semiconductor optical amplifier," J. Opt. Soc. Korea 16, 13-16 (2012).

3. N. K. Dutta and Q. Wang, Semiconductor Optical Amplifiers (World Scientific Pub, 2006).

4. J. E. McGeehan, S. Kumar, and A. E. Willner, "Simultaneous optical digital half-subtraction and-addition using SOAs and a PPLN waveguide," Opt. Express 15, 5543-5549 (2007).

5. J. K. Rakshit, T. Chattopadhyay and J. N. Roy, "Design of micro ring resonator based all optical adder/subtractor," Theoretical and Applied Physics 1, 32-43 (2013).

6. S. K. Chandra, "All optical single module phase encoded half-adder and half-subtractor exploiting four wave mixing in semiconductor optical amplifier," Journal of Electronics and Communication Engineering 6, 67-71 (2013).

7. S. Kaur and R. Kaler, "All optical integrated full addersubtractor and demultiplexer using SOA-based Mach-Zehnder interferometer," International Journal of Engineering Science 4, 303-310 (2012).

8. F. Remacle and R. Levine, "All-optical digital logic: Full addition or subtraction on a three-state system," Physical Review A 73, 033820 (2006).

9. D. K. Gayen, R. K. Pal, and J. N. Roy, "All-optical adder/subtractor based on terahertz optical asymmetric demultiplexer," Chinese Optics Letters 7, 530-533 (2009).

10. C. Gui and J. Wang, "Simultaneous optical half-adder and half-subtracter using a single slot waveguide," IEEE Photonics Journal 5, 684-691 (2013).
11. B. Dai, S. Shimizu, X. Wang, and N. Wada, "Simultaneous all-optical half-adder and half-subtracter based on two semiconductor optical amplifiers," IEEE Photon. Technol. Lett. 25, 91-93 (2013).

12. S. Sahu, R. R. Pal, and S. Dhar, "A novel method of implementing nonlinear material based all-optical binary half subtractor and full subtractor system," J. Electron Devices 10, 493-498 (2011).

13. L. A. Bakhtiar, E. Yaghoubi, A. Adami, S. M. Hamidi, and M. Hosseinzadeh, "The design of half-subtractor logic function based on nonlinear directional coupler," Journal of Computer Engineering 1, 3-11 (2009).

14. H. Sun, Q. Wang, H. Dong, and N. K. Dutta, "XOR performance of a quantum dot semiconductor optical amplifier based Mach-Zehnder interferometer," Opt. Express 13, 18921899 (2005).

15. M. V. D. Poel and J. M. Hvam, "Ultrafast dynamics of quantum-dot semiconductor optical amplifiers," Journal of Materials Science: Materials in Electronics 18, 51-55 (2007).

16. A. Kotb, "NOR gate based on QD-SOA at $250 \mathrm{Gbit} / \mathrm{s}$," Optical and Quantum Electronics 45, 473-480 (2013).

17. Y. B. Ezra, B. I. Lembrikov, and M. Haridim, "Ultrafast all-optical processor based on quantum-dot semiconductor optical amplifiers," IEEE J. Quantum Electron. 45, 34-41 (2009).

18. C. Meuer, J. Kim, M. Laemmlin, S. Liebich, A. Capua, G. Eisenstein, A. R. Kovsh, S. S. Mikhrin, I. L. Krestnikov, and D. Bimberg, "Static gain saturation in quantum dot semiconductor optical amplifiers," Opt. Express 16, 82698279 (2008).

19. A. Rostami, H. A. Nejad, R. M. Qartavol, and H. R. Saghai, "Tb/s optical logic gates based on quantum-dot semiconductor optical amplifiers," IEEE J. Quantum Electron. 46, 354-360 (2010).

20. H. Han, M. Zhang, P. Ye, and F. Zhang, "Parameter design and performance analysis of a ultrafast all-optical XOR gate based on quantum dot semiconductor optical amplifiers in nonlinear mach-zehnder interferometer," Opt. Commun. 281, 5140-5145 (2008).

21. W. Lin, S. Ma, H. Hu, and N. K. Dutta, "All optical latches using quantum-dot semiconductor optical amplifier," Opt. Commun. 285, 5138-5143 (2012).

22. W. Yang, M. Zhang, and P. Ye, "Analysis of $160 \mathrm{~Gb} / \mathrm{s}$ all-optical NRZ-to-RZ data format conversion using quantumdot semiconductor optical amplifiers assisted Mach-Zehnder interferometer," Opt. Commun. 282, 1744-1750 (2009).

23. E. Dimitriadou and K. Zoiros, "On the design of ultrafast all-optical NOT gate using quantum-dot semiconductor optical amplifier-based Mach-Zehnder interferometer," Optics \& Laser Technology 44, 600-607 (2012).

24. E. Dimitriadou and K. Zoiros, "On the feasibility of ultrafast all-optical NAND gate using single quantum-dot semiconductor optical amplifier-based Mach-Zehnder interferometer," Optics \& Laser Technology 44, 1971-1981 (2012).

25. S. Singh, "Ultrahigh speed optical signal processing logic based on an SOA-MZI," IEEE J. Select. Topics Quantum Electron. 18, 970-977 (2012). 